\title{
Les ermites et les reclus(es) : à propos de la figure du maître dans la Queste del Saint Graal, roman arthurien du XIIIe siècle
}

La présence des ermites, des reclus et des recluses dans les textes littéraires du Moyen Âge, qui surprend peut-être le lecteur contemporain, n’étonne pourtant ni les protagonistes ni les destinataires des textes médiévaux. Cette catégorie particulière de héros s'inscrit avec évidence dans le paysage matériel et spirituel de nombreux ouvrages, en l'enrichissant du génie de l'orientale lumen que Guillaume de Saint-Thierry, ami de saint Bernard de Clairvaux et admirateur de la sagesse des Pères du Désert, louait dans la fameuse Lettre aux frères du Mont-Dieu (voir Guillaume de Saint-Thierry, 1975) ${ }^{1}$ adressée à ceux « par qui la lumière de l'Orient et l'antique ferveur religieuse des monastères égyptiens (...) se répandent dans les ténèbres occidentales et dans les froidures des Gaules » (Guillaume de Saint-Thierry, 1975, p. 145)². Cette redécouverte de la tradition érémitique dans la théologie et la spiritualité du XII siècle ne reste pas sans importance pour le développement de la littérature romanesque de l'époque (voir Bretel, 1995). C’est en particulier dans les romans arthuriens français des XII XIII ${ }^{e}$ siècles, écrits en vers ou en prose, que les ermites et les reclus(es) manifestent

- Katarzyna Dybeł - professeur titulaire à l'Institut de Philologie Romane de l'Université Jagellonne de Cracovie. Adresse de correspondance : Instytut Filologii Romańskiej UJ, Al. Mickiewicza 9 A, 31-120 Kraków ; e-mail : katarzyna.dybel@uj.edu.pl ORCID iD : https://orcid.org/0000-0002-6259-732X

1. Déchanet fixe la rédaction de la Lettre aux frères du Mont-Dieu à 1144. Au XIII siècle, Bernard de Clairvaux passe pour l'auteur de cette Lettre. La chartreuse du Mont-Dieu, fondée par Odon (dit parfois Eudes) de Saint-Rémy, dans le diocèse de Reims (la bulle de fondation de l'abbaye est datée du 23 novembre 1136), proclame le retour à l'idéal évangélique des premiers Pères du Désert.

2. La Lettre louait non seulement la puissance de l'orientale lumen, mais aussi la vie des ermites, considérée comme «la plus proche du ciel » (Guillaume de Saint-Thierry, 1975, p. 145) et la perfection de cet état sublime qui « traverse les cieux, s’égale aux anges, imite l’angélique pureté » (p. 155). 
leur présence (et de façon intense) ; ils y deviennent la figure du maitre spirituel, complémentaire à celle du maître chevaleresque. Cet article se propose d'examiner cette présence emblématique et puissamment inspiratrice sur l'exemple de la Queste del Saint Graal, texte anonyme français en prose datant de la première moitié du XIII ${ }^{e}$ siècle, très représentatif de la littérature arthurienne de son époque. Le roman fait partie du Lancelot-Graal, cycle romanesque volumineux dont les branches embrassent : L'Estoire del Saint Graal, Merlin, Lancelot en prose, La Queste del Saint Graal, La Mort le Roi Artu. Le nom du mystérieux "Mestre Gautier Map », cité comme auteur dans les dernières lignes de la Queste del Saint Graal, est considéré aujourd'hui comme une mystification littéraire. De nombreux chercheurs considèrent ce roman comme le fruit d'une production littéraire émanant de moines cisterciens, mais la question des influences et de l'auteur reste ouverte.

La présence des ermites marque la trame narrative de la Queste del Saint Graal de façon intense et efficace. Ils sont ici - plus que les religieux vivant en communauté dans des abbayes - une composante importante du climat spirituel du roman. C'est à eux que l'auteur confie la mission importante de convertir les chevaliers qui en ont besoin, de donner le vrai sens à leurs quêtes et d'expliquer les mystérieuses aventures rencontrées lors de ces quêtes. Ils deviennent ainsi les maîtres spirituels de ceux qui viennent chercher refuge dans leurs ermitages. Les rencontres sont courtes mais efficaces : une nuit, un jour, parfois quelques heures à peine suffisent pour faire subir aux arrivants une métamorphose radicale, une métanoïa profonde qui est ici synonyme de conversion accompagnée de repentance et de pénitence. Les ermites ne sont pas dans ce roman des laïcs, mais des prêtres qui exercent leur mission d'accompagnement d'autrui sur la voie du Salut. De cette façon un ermite anonyme marque la vie de Gauvain lors de sa quête du Saint Graal. Le narrateur précise que cette aventure spirituelle se joue individuellement et il insiste sur le fait que la quête d'un chevalier errant se doit d'être solitaire : «Et a hore de vespres se departirent et tint chascuns sa voie. Et mesire Gauvains chevaucha tant qu'il vint a un hermitage, et trova que li hermites ert en sa chapele et chantoit ses vespres de Nostre Dame. Et il descent de son cheval et les oï, et puis demande l'ostel el nom de sainte charité ; et cil li otroie molt bonement " (La Queste del Saint Graal, 1980, p. 53) [ «À l'heure de vêpres ils se séparèrent. Messire Gauvain alla jusqu'à un ermitage, dont l'ermite disait ses vêpres dans la chapelle. Il descendit de cheval et écouta, puis demanda le gîte qui lui fut courtoisement accordé » (La Quête du Graal, 1965, p. 98)]. Le roman met donc en scène la rencontre de deux personnages solitaires qui vivent leur solitude de manière différente mais complémentaire. Dans la suite du récit, l'ermite est appelé à plusieurs reprises « li preudons » ("le prud'homme ») - aussi bien par le narrateur que par Gauvain. Ce terme souligne l'aspect chevaleresque 
du personnage de l'ermite ${ }^{3}$ qui par le fait d’être prêtre, mais aussi de "semble[r] molt preudons » (La Queste del Saint Graal, 1980, p. 54) [" semble[r] fort prud'homme » (La Quête du Graal, 1965, p. 99)], gagne la confiance de Gauvain qui se confesse à lui. La confession du neveu du roi Arthur semble annoncer la suite heureuse de la quête pour Gauvain. Le narrateur fait valoir la disponibilité de l'ermite, prêt à servir en prodiguant ses conseils, mais aussi respectueux de la liberté d'agir du chevalier. L'ermite ne force aucunement Gauvain, il l'encourage à se confesser. Dès qu'il apprend le nom du chevalier, il lui déclare : "Certes, sire, s'il vos plaisoit, je voldroie mout savoir de vostre estre " (La Queste del Saint Graal, 1980, p. 53) ["Certes, messire (...), je voudrais bien s'il vous plaisait, aller au fond de votre âme » (La Quête du Graal, 1965, p. 99)]. Gauvain répond avec franchise à cette invitation, en avouant sa volonté de se confesser, mais aussi en y mettant une condition : celle d'obtenir - de la part de l'ermite - l'explication d'une aventure qui lui est arrivée. La confession faite, l'ermite adopte un ton sérieux, en reprochant à Gauvain d'avoir mené «la plus orde vie et la plus mauvese que onques chevaliers menast " (La Queste del Saint Graal, 1980, p. 54) [" la vie la plus déréglée et la plus mauvaise que mena jamais chevalier » (La Quête du Graal, 1965, p. 99)] et le manque de miséricorde envers sept chevaliers frères tués avec le concours de Gauvain. Immédiatement après, il lui parle de la Miséricorde divine, en l'invitant à entrer en pénitence. De nouveau, il conseille, il encourage, sans imposer de conduite, mais aussi sans cacher la gravité de l'état de l'âme de Gauvain. La forme du conditionnel utilisée dans son discours souligne ce désir de sauver son hôte : "se tu vouloies lessier ceste mauvese vie que tu as ja si longuement maintenue, encore te porroies tu acorder a Nostre Seignor " (La Queste del Saint Graal, 1980, p. 55) [ " si tu voulais laisser cette vie mauvaise que tu as menée si longtemps, tu pourrais encore retrouver Dieu » (La Quête du Graal, 1965, p. 100)]. Ce n'est pas : «tu dois laisser ", « laisse », mais : « si tu voulais ». Quand Gauvain refuse la pénitence, l'ermite l'accepte et le narrateur conclut brièvement : «Et li preudome le let a tant, que plus ne li dit, car il voit bien que ses amonestemenz seroit peine perdue " (La Queste del Saint Graal, 1980, p. 55) [ "Et le prud'homme le laisse, car il voit bien que ses exhortations ne sont que peine perdue » (La Quête du Graal, 1965, p. 100)]. Cette réaction témoigne de la clairvoyance, du don de discernement de l'ermite. Ses conseils refusés, il laisse partir le chevalier et le texte se tait sur la suite de cette rencontre. On ne peut que supposer que « le preudome " va entourer le partant de sa prière, comme les ermites romanesques avaient l'habitude de faire. Dans l'épisode évoqué le maitre spirituel subit un échec, mais le texte souligne nettement que la faute est au disciple aveuglé par ses passions et trop faible pour choisir

3. La même fusion du langage religieux et courtois est à observer dans la scène de la première rencontre de Lancelot avec Galaad qui a lieu dans une abbaye de religieuses qui élèvent Galaad. Les religieuses sont appelées «nonains », mais aussi « dames » (voir La Queste del Saint Graal, 1980, p. 2). 
pleinement la voie de la conversion ${ }^{4}$. La confession s'avère ici un seuil que Gauvain franchit certes, mais non pour avancer : il recule au contraire, pour reprendre de plus belle le chemin d'autrefois condamné par l'ermite.

Le personnage de l'ermite manifeste aussi sa présence dans la partie de la Queste del Saint Graal qui relate les aventures de Lancelot. Cette rencontre est marquée par une tonalité bien plus dramatique. L'amant de la reine Guenièvre, écrasé psychiquement après son échec lors de la manifestation du Saint Graal dans la forêt et privé du cheval, du heaume et de l'épée, arrive - "mornes et pensis et tant dolenz que nus plus" (La Queste del Saint Graal, 1980, p. 62) [ " tout pensif et morne et dolent " (La Quête du Graal, 1965, p. 107)] - à l'heure de prime, dans un ermitage situé sur un tertre. Dans la suite du récit nous apprenons que l'ermite est prêtre : il célèbre la messe, écoute la confession, impose la pénitence et donne l'absolution ${ }^{5}$. Tout comme dans l'épisode de la visite de Gauvain à l'ermitage, la narration est centrée autour du motif de la confession. Lancelot désire se confesser et c'est dans cet acte de volonté que se dessine la grande différence entre les deux chevaliers. Gauvain a accepté de se confesser, encouragé par l'ermite, tandis que Lancelot de sa propre initiative a demandé la confession : il appelle l'ermite, le tire à part et lui demande de le conseiller. Le texte souligne, une fois de plus, le don du conseil associé au personnage de l'ermite. Celui-ci réagit d'une façon qui révèle son habitude de rendre ce service spirituel aux chevaliers errants : «Et li preudons li demande : 'De quoi volez vos conseil ? Est ce de confession ?' - 'Sire, oil', fet il. - 'Et de par Nostre Seignor', fait li preudons " (La Queste del Saint Graal, 1980, p. 63) ["Le prud'homme lui demanda : 'Quel conseil voulez-vous ? Est-ce confession ?' - 'Oui, Sire. - Ainsi soit-il', dit l'ermite " (La Quête du Graal, 1965, p. 107)]. La scène de cette rencontre devient prétexte à expliquer le sens et l'importance du sacrement de la confession, faisant référence à la doctrine de la gratuité de l'Amour et de la Miséricorde divine, du sacrifice salvateur du Christ. La confession est ainsi montrée comme canal de la grâce, comme source d'une force surnaturelle qui seule est capable de relever l'homme et de lui permettre de vaincre sa faiblesse. L'ermite précise également son propre rôle dans la confession : il est médiateur, témoin et ministre de la grâce, mais le vrai destinataire de la confession c'est Nostre Seigneur : « Et dites orendroit vostre estre et vostre afere a lui en audience devant moi, et je vos aiderai a secorre a mon pooir, et vos conseillerai de quan que je porrai » (La Queste del Saint Graal, 1980, p. 65) [" Dites-lui maintenant votre histoire en confession devant moi ; je vous aiderai selon mon pouvoir, et vous conseillerai », dit-il à Lancelot (La Quête du Graal, 1965, p. 109)]. Il rappelle en même temps les conditions pour obtenir le pardon et la Miséricorde : "veraie

4. On peut percevoir dans cet épisode un écho lointain de l'épisode de la rencontre de Tristan et Yseut avec l'ermite Ogrin, relaté dans la version de Béroul de Tristan et Yseut. L'effort de la conversion dépasse les deux amants qui le refusent au nom de leur amour.

5. Le narrateur précise que, lors de la messe, il est accompagné de " son clerc » (La Queste del Saint Graal, 1980, p. 62) qui chante avec lui la messe. 
confession de bouche et de repentance de cuer et en amendement de vie " (La Queste del Saint Graal, 1980, p. 65) ["vraie confession de bouche et repentance de cœur et amendement de vie" (La Quête du Graal, 1965, p. 109)]. La confession - qui est ici une confession générale, embrassant toute la vie de Lancelot - adopte la forme d'un long dialogue fait sur un ton amical et en même temps fort exigeant. Le texte souligne à plusieurs reprises la nécessité de la confiance de celui qui se confesse en celui qui écoute la confession - le prêtre qui représente de façon visible le Christ invisible. Avant d'avouer son péché, Lancelot «pense un petit, come cil qui onques ne reconut l'afere de lui et de la reine " (La Queste del Saint Graal, 1980, p. 65) ["réfléchit un instant ; jamais il n'a avoué ce qui s'est passé entre la Reine et lui » (La Quête $d u$ Graal, 1965, p. 110)]. La confiance gagne, mais il n'y va point d'une simple sympathie portée à l'ermite : c'est un acte de foi du protagoniste par lequel il exprime la conviction que sa confession est écoutée par Nostre Seigneur en personne. Lors de la confession l'ermite agit comme un guide sûr, expérimenté et exigeant : il parle avec sûreté, en expliquant à Lancelot le sens caché des aventures et en les interprétant à la lumière des textes des Évangiles. Son autorité, due à la conformité de son enseignement aux paroles de Dieu, aide Lancelot à prendre la décision de se convertir et de prononcer les paroles fermes et courageuses : «Sire, fet Lancelot, je le les, en tel maniere que ja mes ne pecherai en li ne en autre " (La Queste del Saint Graal, 1980, p. 71) [ «Sire, fit Lancelot, jamais je ne commettrai plus ce péché ni avec la Reine ni avec une autre » (La Quête du Graal, 1965, p. 114)]. La réaction de l'ermite est simple et transparente - le narrateur relate : «Et quant li preudons l'ot, si li enjoint tel penitance com il cuide que il puisse fere, et l'asoult et beneist et li prie que il remaigne hui mes o lui » (La Queste del Saint Graal, 1980, p. 71) [ Q Quand le prud'homme l'entendit, il lui imposa une pénitence, lui donna l'absolution et sa bénédiction, puis le pria de demeurer aujourd'hui en sa compagnie » (La Quête du Graal, 1965, p. 114)]. Lors de ce court séjour l'ermite travaille encore à renforcer les fruits de cette conversion : « Einsi demora Lancelot o le preudome qui l'amonestoit de bien fere. Et tant li dist li hermites bones paroles que Lancelot se repent mout de la vie qu'il a si longuement menee » (La Queste del Saint Graal, 1980, p. 71) [ «Lancelot demeura donc, et l'ermite l'admonesta tant et si bien qu'il eut grande repentance de sa vie passée " (La Quête $d u$ Graal, 1965, p. 114)], continue le narrateur. L'épisode s'achève sur les mots qui informent le destinataire du texte de la contrition sincère de Lancelot et de la promesse faite «en son cœur » de ne plus retomber dans le péché. Ainsi toutes les conditions de la confession, telles qu'elles étaient précisées par la doctrine de la confession de l'époque, sont-elles remplies. L'ermite a réussi dans son rôle de guide spirituel.

Le même texte met en scène lépisode de la rencontre d'un autre protagoniste-chevalier, Perceval, avec une recluse à qui il doit une partie importante de sa formation morale et spirituelle. Il faut rappeler que la présence des recluses dans les textes romanesques du XIII ${ }^{e}$ siècle n'est pas rare. Elle reflète la réalité historique de cette époque où les femmes jouaient un rôle bien plus considérable qu'on ne le pense aujourd'hui, en assumant des responsabilités non seulement politiques mais aussi spirituelles. 
La réclusion était une forme de vie ascétique sévère (dont les origines remontent aux pratiques des moines syriens au IV ${ }^{e}$ siècle) qu'il ne faut pas confondre avec l'érémitisme; elle était choisie plus souvent par les femmes que par les hommes. Les recluses habitaient de coutume dans des bâtiments adjacents aux monastères ou aux autres lieux de culte, ce qui leur permettait d'assister fréquemment à la liturgie (Viller M., Long R. J., 1987, p. 218). Réputées pour leur sagesse et leurs charismes multiples, elles attiraient ceux qui cherchaient la guérison physique ou spirituelle. Au XIII ${ }^{e}$ siècle, la réclusion était devenue moins fréquente dans les sociétés occidentales, ce qui rend d'autant plus surprenant le fait que le roman arthurien en France fait d'elle un motif important qui marque un espace privilégié pour ces rencontres qui orientent la narration et donnent leur sens aux quêtes chevaleresques.

La recluse qui accueille Perceval s'avère être une de ses tantes. Le texte ne dit pas son nom, mais il explique qu'autrefois elle était appelée « Reine de la Terre Gaste » $\mathrm{La}$ Queste del Saint Graal, 1980, p. 73). Elle mène une vie typique des recluses de l'époque, ce qu'elle explique personnellement à son neveu :

Si pris maintenant grant partie de mon avoir, et m'en afoi en si sauvage leu, por ce que je ne fusse trovee maintenant ; et fis fere cest reclus et ceste meson tele come vos la veez, et i mis o moi mon chapelain et ma mesniee, et entrai en cest reclus en tel maniere que ja mes, se Dieu plest, n'en istrai tant com je vive, ainz morrai ou servise Nostre Seignor et userai le remanant de ma vie. (La Queste del Saint Graal, 1980, p. 80-81)

[Je pris donc une grande part de mes biens et m'en allai dans cette solitude pour qu'on ne pût me trouver; je fis bâtir cet ermitage, où jétablis mon chapelain et mes gens, et s'il plait à Dieu, je n'en sortirai pas vivante, mais userai le reste de ma vie et mourrai au service de Notre Seigneur. (La Quête du Graal, 1965, p. 125)]

Contrairement à Perceval qui, dans le Conte du Graal de Chrétien de Troyes, s'est rendu chez l'ermite pour trouver de l'aide dans son état de mélancolie, le protagoniste de la Queste del Saint Graal se rend chez la recluse pour obtenir des nouvelles d'un chevalier mystérieux qu'il désire rencontrer : «Or dit li contes que quant Perceval se fu partiz de Lancelot, qu'il retorna a la recluse dont il cuidoit oïr noveles dou chevalier qui eschapez lor estoit » (La Queste del Saint Graal, 1980, p. 71-72) [ "Alors dit le conte que quand Perceval eut quitté Lancelot, il retourna chez la recluse pour avoir des nouvelles du chevalier qui leur avait échappé » (La Quête du Graal, 1965, p. 117)]. En outre, Perceval espère y trouver un refuge pour la nuit qui commence à tomber. Il ne prévoit pas qu'il y trouvera un maitre qui lui révélera les secrets du Saint Graal. La conversation qui suit s'organise autour du sens de la quête entreprise par Perceval. La recluse, stimulée par les questions de son neveu, se lance dans un long exposé, centré autour du motif des trois tables liées au Saint Graal et du motif du chevalier désiré - du "Bon Chevalier ", "Verais Chevalier» (La Queste del Saint Graal, 1980, p. 77) que les autres chevaliers doivent « tenir a mestre et a pas- 
tor " (La Queste del Saint Graal, 1980, p. 78) [ " considérer comme maître et pasteur » (La Quête du Graal, 1965, p. 122)] et qui, annoncé par les prophéties lointaines, doit accomplir les merveilles du Graal. La recluse adopte un ton sérieux et solennel adapté au contenu du discours - il y va des choses sacrées dont le sens et les origines sont révélés uniquement aux élus. Elle devient alors un maitre qui initie son disciple au monde secret des merveilles du Saint Graal :

Vos savez bien que puis l'avenement Jhesucrist a eu trois principaus tables ou monde. La premiere fu la Table Jhesucrit ou li apostre mengierent par plusor foiz. Cele fu la table qui sostenait les cors et les ames de la viande dou Ciel. A cele table sistrent li frere qui estoient une meisme chose en cuer et en ame (...). Et icele table establi li Aigniax sanz tache qui fu sacrefiez por nostre redemption. Aprés cele table fu une autre table en semblance et en remembrance de lui. Ce fu la Table dou Saint Graal, dont si grant miracle furent jadis veu en cest païs au tens Joseph d'Arimacie, au comencement que crestientez fu aportee en ceste terre, que tuit preudome et tuit mescreant devroient avoir toz jorz mes celui miracle en remenbrance. (...) Aprés cele table fu la Table Reonde par le conseil Merlin, qui ne fu pas establie sans grant senefiance. Car en ce qu'ele est apelee Table Reonde est entendue la reondece del monde et la circonstance des planetes et des elemenz el firmament; et es circonstances dou firmament voit len les estoiles et mainte autre chose ; dont len puet dire que en la Table Reonde est li mondes senefiez a droit. Car vos poez veoir que de toutes terres ou chevalerie repere, soit de crestienté ou de paiennie, viennent a la Table Reonde li chevalier. Et quant Diex lor en done tel grace qu'il en sont compaignon, il s'en tienent a plus boneuré que s'il avoient tout le monde gaangnié, et bien voit len que il en lessent lor peres et lor meres et lor fames et lor enfanz. (La Queste del Saint Graal, 1980, p. 74-77)

[Vous savez que depuis l'avènement de Jésus-Christ il y eut trois tables principales dans le monde. La première fut la Table de Jésus-Christ où les apôtres mangèrent plusieurs fois. Ce fut la table qui soutenait les corps et les âmes de la nourriture du Ciel. À cette table s'assirent les frères qui n'étaient qu'un, de cœur et d'âme (...). Et cette table fut instituée par l'Agneau sans tache qui fut sacrifié pour notre rédemption. Après cette table, il y en eut une autre à la semblance et remembrance de la première. Ce fut la Table du Saint-Graal, dont on vit de si grands miracles en ce pays au temps de Joseph d'Arimathie, au commencement de la Chrétienté sur la terre. Tous, prud'hommes et mécréants, devraient se souvenir de ce miracle. (...) Après cette table, il y eut encore la Table Ronde établie selon le conseil de Merlin et pour une grande signifiance. On l'appelle Table Ronde pour désigner par là la rondeur du monde, et le cours des planètes et des astres au firmament ; dans les révolutions célestes on voit les étoiles et mainte autre chose, aussi peut-on dire que la Table Ronde représente bien le monde. Vous voyez bien que de toutes les terres où habite la chevalerie, soit chrétiennes, soit païennes, les chevaliers viennent à la Table Ronde. Quand Dieu leur donne la grâce d'en être compagnons, ils s'en tiennent plus honorés que s'ils avaient conquis le monde entier, et ils quittent pour cela pères et mères, femmes et enfants. (La Quête du Graal, 1965, p. 119-120)] 
La figure de recluse se confond ici avec celle de philosophe et de théologien : elle recourt aux arguments d'ordre cosmologique, mais surtout évangélique et théologique, en interprétant l'histoire du Saint Graal dans le contexte sotériologique et en proposant une sorte d'exégèse du Graal. En fait, cet entretien avec Perceval fait penser aux disputes médiévales - pratiquées dans les écoles monastiques ou cathédrales et basées sur le schéma quaeritur - respondetur - dans lesquelles la question et la réponse devenaient un prétexte pour présenter un exposé doctrinal sur des questions données, en particulier celles de théologie trinitaire, christologique ou sacrementaire. De ce point de vue, l'œuvre littéraire se présente comme locus theologicus - lieu de rencontre de la matière littéraire avec l'intuition spirituelle et la doctrine théologique. Le langage de la recluse est marqué par l'emploi des substantifs " allégorie ", «symbole » (confondus d'ailleurs au Moyen Âge), "senefiance » et des verbes " annoncer ", "senefier » qui renforcent l'aspect exégétique de ce passage. D’autre part, ce langage est austère, il est fait " pour découvrir le vrai plutôt que pour l'orner ou le suggérer " (Béguin, 1963, p. 39).

La recluse parle et Perceval écoute. Cette scène évoque par excellence la figure du maître et du disciple, connue des autres textes arthuriens de cette période, où le maître induit son disciple à découvrir son identité, tout en le préparant au rôle que la Providence lui fera jouer. La présence de la recluse qui provoque le ralentissement de l'action, faisant découvrir à Perceval et au destinataire du texte un message nouveau, démontre également que l'univers des maitres romanesques n'est pas dominé par les hommes : les femmes y tiennent également une place importante. Ce personnage subtil, associé comme celui de l'ermite à la dimension sapientiale du texte, dévoile un type de raisonnement complexe où la voix masculine propre au théologien se confond avec la voix féminine qui fait avancer le disciple dans la sagesse du cœur et de l'esprit.

La présence des ermites et des reclus(es), signes de stabilité, peut sembler peu compatible avec la mobilité de l'univers de la chevalerie arthurienne. Pourtant, il faut envisager cette relation bien plus dans l'optique de la complémentarité que dans celle de l'opposition - complémentarité qui jette un pont entre les chevaleries céleste et terrestre, entre les sagesses intellectuelle et spirituelle. Si les abbayes sont dans la Queste del Saint Graal le lieu de l'éducation chevaleresque, de la formation prolongée (Galaad est élevé dans une abbaye de femmes et il y séjourne jusqu'à son adoubement et son départ à la cour du roi Arthur), de l'hospitalité ou des soins apportés aux blessures, les ermitages sont pour leur part un lieu d'évolution spirituelle, d'autotransgression, de conversion ; ils sont plus que tout autre espace signe de l'Éternité.

Dans le texte étudié, les ermites ne sont pas accompagnés de disciples qui les suivraient continuellement, à l'exemple des abbas orientaux entourés de leurs fils spirituels. Leur activité reste au contraire marquée du sceau de la solitude et de l'hospita- 
lité qui font d'eux des personnages disponibles à tout arrivant envers qui ils n'hésitent jamais à exercer leur charisme de guide spirituel. Si la mission du maître est de faire avancer le disciple, les ermites la remplissent de façon exemplaire. On remarquera que la vision du monde telle qu'elle apparait dans la Queste del Saint Graal manifeste une grande estime à l'égard de cette catégorie de héros. Même si dans certains épisodes l'ermite et la recluse ne sont pas présentés directement en action, les commentaires faits à leur propos ne laissent guère de doutes quant au rôle de maîtres qui leur est accordé dans le roman. Les protagonistes et le narrateur parlent d'eux avec le plus grand respect et ce respect permet de voir en eux les guides spirituels des personnages fictifs mais aussi des lecteurs. Il semble particulièrement significatif que les ermites apparaissent privés de leurs noms. Ne pourrait-on voir dans ce fait un accent qui souligne leur humilité et qui concentre l'intérêt des épisodes dans lesquels ils interviennent sur leur mission et non pas sur leurs personnalités ? Ils s'effacent devant le rôle qu'ils ont à jouer.

Comme le fait remarquer Albert Béguin, la Queste del Saint Graal est « un authentique chef-d'œuvre littéraire, l'un des plus beaux de tout le moyen âge. Il n'a rien d'une prédication ou d'un récit seulement édifiant ; les sermons même des ermites qui périodiquement instruisent les héros et leur expliquent leurs songes ou leurs aventures, ne rompent pas la trame de l'invention romanesque " (Béguin, 1963, p. 38). Ces ermites séjournent de façon unique dans « un monde de forêts, de vallons et de rivières, jalonné de châteaux aux fantastiques prestiges, où des chevaliers errants cherchaient à longueur d'année aventure, rencontrant de temps à autre aussi bien des ermites avertis du sens des songes que de hardies et savantes demoiselles, bizarrement solitaires » (Bonnefoy, 1965, p. 10). L'univers de la Queste del Saint Graal, qui pourrait sembler tellement cohérent et harmonieux, est pourtant déchiré par des contrastes rendus à merveille dans les textes arthuriens. Comme le soutient Albert Béguin, au Moyen Âge la légende du Graal « avait revêtu des formes très diverses, en lesquelles se reflète l'un des plus grands conflits spirituels qu'ait traversés la civilisation européenne » (Béguin, 1963, p. 25). Les personnages de l'ermite et de la recluse ont une capacité unique d'adoucir ces conflits, de les pacifier. En même temps, leur présence est importante du point de vue de la construction de la trame romanesque : elle influe sur le rythme de la quête chevaleresque, en le ralentissant et en faisant scruter le sens et le but de la quête, mais aussi de la mission chevaleresque. Ces figures emblématiques du maître, accompagnées des personnages symétriques et complémentaires que sont les disciples, s'avèrent porteuses d'un message anthropologique important, en véhiculant une parole métaphysique et eschatologique sur l'homme et sa destinée, qui dépasse les limites de la fiction littéraire. Elles témoignent d'un courant visant à rechristianiser une société courtoise, à respiritualiser le monde profane auquel l'auteur et ses destinataires devaient être confrontés. Il y va d'une littérature mise au service de l'évangélisation. Maîtres par la parole, qui renseignent et enseignent, maîtres par le silence et la solitude, et surtout maîtres par la prière, les ermites et les reclus(es) se révèlent comme des êtres "vivant sur terre, mais demeurant dans les cieux " 
(Guillaume de Saint-Thierry, 1975, p. 269) dont la vie est une sorte de vie angélique. Conduits par l'Esprit, ils conduisent vers cet Esprit ceux qui frappent à leurs portes. Les paroles de ces prophètes d'un monde à venir deviennent la force motrice de la quête de Dieu (que symbolise de fait la quête du Saint Graal) dans laquelle s'engagent les meilleurs chevaliers du monde arthurien.

\section{RÉFÉRENCES}

Texte étudié :

La Queste del Saint Graal. Roman du XIII siècle. (1980). A. Pauphilet (éd.). $2^{\text {e }}$ tirage. Paris : Champion.

\section{Textes consultés :}

Guillaume de Saint-Thierry. (1975). Lettre aux frères du Mont-Dieu. J. Déchanet (intr., texte crit., trad. et notes). Paris : Éditions du Cerf.

La Quête du Graal. (1965). A. Béguin, Y. Bonnefoy (éd.). Paris : Éditions du Seuil.

\section{Ouvrages critiques :}

Béguin, A. (1965). Préface. Dans A. Béguin, Y. Bonnefoy (éd.). La Quête du Graal (p. 25-43). Paris : Éditions du Seuil.

Bonnefoy, Y. (1965). Les romans arthuriens et la légende du Graal. Dans A. Béguin, Y. Bonnefoy (éd). La Quête du Graal (p. 9-21). Paris : Éditions du Seuil.

Bretel, P. (1995). Les ermites et les moines dans la littérature française du moyen âge (11501250). Paris : Champion.

Tudor, A. P. (2006). Érémitisme et solitude dans la première Vie des Pères. Le Moyen Âge, tome CXII(1), 43-61. Récupéré de https://www.cairn.info/revue-le-moyen-age-2006-1page-43.htm

Généralités :

Viller, M., Long, R. J. (1987). Dictionnaire de spiritualité, vol. XIII. Paris : G. Beauchesne et ses fils.

RÉSUMÉ : L'article propose une étude approfondie et interdisciplinaire de deux types de personnages emblématiques et inspirateurs qui manifestent leur présence dans la Queste del Saint Graal, texte arthurien français en prose du XIII ${ }^{\mathrm{e}}$ siècle : le personnage de l'ermite et celui de la recluse. Figures du maître par excellence, ils sont porteurs du savoir et de la sagesse qui dépassent largement le cadre d'un simple didactisme et de la parénèse. Leur présence témoigne de l'ouverture de la littérature occidentale au souffle puissant de la spiritualité du christianisme oriental. Elle importe également pour la construction et le sens de l'univers représenté de l'ouvrage analysé.

Mots-clés : La Queste del Saint Graal, roman arthurien, figure du maître, ermite, recluse 
Hermits and Recluses: on the figure of master in the 13th century Arthurian romance, The Quest for the Holy Grail

ABSTRACT: The article analyses the figures of hermits and recluses presented in The Quest for the Holy Grail, and functioning in this anonymous Arthurian romance of the 13th century as variants of the figure of a spiritual mentor, complementing the figure of a master in chivalry. These characters shape the narrative discourse and enrich the sapiential, moral and formative aspects of the work. They also re-define the courtly ethos by introducing the idea of celestial chivalry, which complements the idea of terrestrial chivalry, typical of the Arthurian romance of the 12th century.

Keywords: The Quest for the Holy Grail, Arthurian romance, figure of master, hermit, recluse 